\title{
Halbleiternanopartikel-modifizierte Elektrode zum Nachweis von Substraten von NADH-abhängigen Enzymreaktionen
}

\author{
Kirsten Schubert, Waqas Khalid, Zhao Yue, Wolfgang J. Parak, Fred Lisdat
}

Zusammenfassung

Es wurde ein Elektrodensystem entwickelt, das aufbauend auf Halbleiternanopartikeln (so genannte Quantenpunkte) die sensitive Detektion des Enzymkofaktors $\mathrm{NADH}$ (nicotinamide adenine dinucleotide) erlaubt. Kolloidale halbleitende CdSe/ZnS-Nanokristalle sind durch ein Dithiol über Chemisorption an Gold gebunden. Das Stromsignal kann durch die Beleuchtung der Quantenpunktmodifizierten Oberfläche beeinflusst werden. Durch Photoanregung entstehen Elektron-LochPaare in den Nanopartikeln, die als anodischer oder kathodischer Photostrom detektiert werden können. Die Immobilisierung der Nanokristalle ist durch amperometrische Photostrom- und Quarzmikrowaage-Messungen (quartz crystal microbalance) verifiziert.

Diese Studie zeigt, dass CdSe/ZnS-Quantenpunktmodifizierte Elektroden eine konzentrationsabhängige $\mathrm{NADH}$-Detektion im Bereich von $20 \mu \mathrm{M}$ bis $2 \mathrm{mM}$ bei relativ niedrigem Potential (um OV vs $\mathrm{Ag} / \mathrm{AgCl}, 1 \mathrm{M} \mathrm{KCl}$ ) ermöglichen. Somit können solche Elektroden in Kombination mit NADH-produzierenden Reaktionen für die lichtgesteuerte Analyse der entsprechenden Substrate des Biokatalysators genutzt werden. Es wird gezeigt, dass mit einem solchen Elektrodensystem und Photostrommessungen ein Glukosenachweis möglich ist.

\section{Abstract}

An electrode system based on semiconductive nanoparticles (so called quantum dots) was developed which allows the sensitive detection of the enzyme cofactor NADH (nicotinamide adenine dinucleotide). Colloidal semiconductive CdSe/ZnS nanocrystals are bound to gold via a dithiol compound by chemisorption. The current signal can be influenced by illumination of the quantum dot-modified electrode surface. Because of photoexcitation electron-holepairs are generated in the nanoparticles which can be detected as anodic or cathodic photocurrent. The immobilisation of the nanocrystals is verified by photocurrent and quarz crystal microbalance (QCM) measurements.

This study shows that $\mathrm{CdSe} / \mathrm{ZnS}$-quatum dot-modified electrodes provide a concentration-dependent detection of $\mathrm{NADH}$ in the range of $20 \mu \mathrm{M}$ up to $2 \mathrm{mM}$ at relatively low overpotentials (around OV vs Ag/ $\mathrm{AgCl}, 1 \mathrm{M} \mathrm{KCl}$ ). Such electrodes can be used in combination with NADH-producing reactions for the lighttriggered analysis of the corresponding substrate of the biocatalyst. The detection of glucose with such an electrode system and photocurrent measurements is shown.

\section{Einleitung}

Die Nanostrukturierung von Oberflächen ist schon seit einigen Jahren ein intensiv untersuchtes Wissenschaftsfeld. Nanostrukturierte Bausteine können verbesserte Eigenschaften wie erhöhte katalytische Aktivität oder Sensitivität aufweisen. (Katz et al. 2004; Wang 2005) Kleine und definierte Strukturen können durch verschiedene Ansätze erreicht werden, z. B. durch molekulare Selbstorganisation, dünne Polymerschichten und/oder Nanopartikel. (Lisdat et al. 2009; Willner et al. 2007; Xiao et al. 2008)
Wegen ihrer Größe besitzen Nanopartikel andere physikalische Eigenschaften im Vergleich zu ihren Volumenmaterialien. Daher kommen sie zunehmend in analytischen Detektionssystemen und Sensoren zur Anwendung. (Katz et al. 2004; Wang 2005; Willner et al.2007; Lin et al. 2007; Gill et al. 2008) Neben metallischen Nanopartikeln und Kohlenstoffnanoröhren haben auch halbleitende Nanopartikel erhebliches Interesse erweckt. Aufgrund ihrer einzigartigen photophysikalischen Eigenschaften sind solche Quantenpunkte als optische Marker in der Biosensorik geeignet. 
Sie besitzen hohe Fluoreszenz-Quantenausbeute, größenabhängige Lumineszenzeigenschaften und eine erheblich größere Stabilität gegen Photobleichung im Vergleich zu organischen Fluorophoren. (Chan et al. 2002; Brus 1991) Verschiedene Synthesewege und chemische Oberflächenmodifizierungen mit unterschiedlichen Liganden ermöglichen die effektive Kopplung von Quantenpunkten mit Biomolekülen und Oberflächen und zur Entwicklung von Hybridsystemen. (Gill et al. 2008; Chan et al. 2002; Pellegrino et al. 2005) Diese Merkmale und die ähnlichen Ausmaße von halbleitenden Nanokristallen im Vergleich zu Biomolekülen haben zur Anwendung als photonische Marker in bioanalytischen Systemen geführt. Somit wurden Quantenpunkte schon in vielfältigen biologischen Erkennungssystemen (Klostranec et al. 2006) verwendet, wie z. B. Immunoassays (Goldman et al. 2004) oder die Detektion von Nukleinsäuren. (Gerion et al. 2003)

Halbleitende Nanokristalle haben außerdem einzigartige elektronische Eigenschaften. Photogenerierte Exzitonen können mit Elektrodenoberflächen elektrisch kommunizieren, was zu einem anodischen oder kathodischen Photostrom führt. Angeregte Leitungsband-Elektronen der Quantenpunkte können zur Elektrode oder einem Elektronenakzeptor in Lösung transportiert werden. Elektronen können ebenso von einer Elektrode oder einem gelösten Elektronendonor zu Valenzbandlöchern in Quantenpunkten transferiert werden. Daher kann eine Quantenpunktschicht zwischen der Elektrode und einem Redoxsystem für eine lichtgesteuerte Auslese der Elektronentransferreaktionen mit der Elektrode genutzt werden. Bis jetzt wurden nur wenige solcher Systeme entwickelt und untersucht. Ein Beispiel sind Quantenpunkt-DNA-Hybridsysteme für die DNA-Detektion und die Konstruktion von organisierten Nanostrukturen. (Katz et al. 2004; Willner et al. 2001; Freeman et al. 2007) Die Interaktion von immobilisierten halbleitenden Nanokristallen mit Proteinen wurde ebenfalls schon in einigen elektrochemischen Studien gezeigt. Die elektrochemische Reaktion von oberflächenfixierten Quantenpunkten mit redoxaktiven Proteinen wie Cytochrom $c$ kann den Photostrom beeinflussen, wie in einer unserer vorherigen Arbeiten gezeigt wurde. (Stoll et al. 2006) Zusätzlich wird dieser Photostrom durch die Interaktion von Cytochrom $c$ mit Superoxidradikalen (Stoll et al. 2008) oder auch Laktatdehydrogenase und Nitratdehydrogenase in Lösung (Katz et al. 2006) kontrolliert. Eine enzymatische Reaktion kann auch durch die Detektion des katalytischen
Produktes an der nanopartikelmodifizierten Elektrode beobachtet werden. (Pardo-Yissar et al. 2003)

Die Beteiligung von NADH bei Reaktionen von über 300 Dehydrogenasen macht es zu einem interessanten Kandidaten für die amperometrische Verfolgung dieser Reaktionen. (Katakis et al. 1997) Seine elektrochemische Detektion kann als Grundlage für die Entwicklung von elektrochemischen Biosensoren genutzt werden. Das Redoxpotential von NADH/NAD ${ }^{+}$liegt bei $-0.32 \mathrm{~V}$ vs SHE. Für die elektrochemische Oxidation von NADH an unbehandelten Elektroden ist eine Überspannung von mehr als $1 \mathrm{~V}$ notwendig. (Gorton 1986) In diesem Potentialbereich sind aber auch viele andere redoxaktive Substanzen oxidierbar. Ein weiteres großes Problem ist die Vergiftung der Elektrodenoberfläche während des Oxidationsprozesses von NADH aufgrund der Bildung von Dimeren oder stabiler Addukte mit den Reaktionsintermediaten. (Jaegfeldt 1981) Um diesen Problemen zu begegnen, wurden verschiedene Methoden zur Vorbehandlung der Elektrodenoberfläche entwickelt. (Blaedel et al. 1975; Lau et al. 2005; Nagy et al. 1995; Munteanu et al. 2002) Eine viel versprechende Variante ist die Modifikation der Oberfläche mit niedermolekularen Mediatoren wie mono-oder polyaromatische Verbindungen. (Katakis et al. 1997; Persson et al. 1990; Prodromidis et al. 2002; Fukuzumi et al. 1984) In neueren Studien wurde die Verwendung von Nanopartikeln als Elektrokatalysator für die Oxidation von NADH demonstriert. (Raj et al. 2005)

In dieser Arbeit wollen wir die Anwendungsmöglichkeit von Quantenpunkten für den NADH-Nachweis zeigen - basierend auf der Immobilisierung von CdSe/ZnSNanokristallen auf Goldelektroden. Die Quantenpunkte bilden auf der Elektrode eine photoschaltbare Zwischenschicht, die die räumliche Auslese der Sensoroberfläche ermöglicht. Das bietet weiterhin die Basis für die Kombination der CdSe/ZnS-Elektrode mit NADH-produzierenden Enzymreaktionen für die lichtgesteuerte Detektion der entsprechenden Enzymsubstrate. Es wird gezeigt, dass mit solch einem Elektrodensystem eine Glukosedetektion durch Photostrommessungen möglich ist.

\section{Experimenteller Teil}

\section{Materialien}

1,4-Benzendithiol (BDT) wurde von Alfa Aesar (Karlsruhe, Dtl.) bezogen. Chloroform, Toluen, Methanol, alle Puffersalze, Pyrroloquinolinquinon (PQQ), N-Ethyl- 
$N^{\prime}$-(3dimethylaminopropyl)carbodiimidhydrochlorid (EDC) und Glukosedehydrogenase (EC 1.1.1.47, GDH) aus Pseudomonas sp. wurden bei Sigma-Aldrich (Taufkirchen, Dtl.) gekauft und ohne weitere Reinigung verwendet. Alle wässrigen Lösungen wurden mit destilliertem Wasser hergestellt (18 M $\Omega$, Millipore GmbH, Dtl.).

Die CdSe/ZnS-Nanopartikel wurden gemäß einer zuvor schon beschriebener Prozedur synthetisiert. (Reiss et al. 2002; Dabbousi et al. 1997) Nach der Synthese wurden die Quantenpunkte mit Trioctylphosphinoxid (TOPO) belegt und in Chloroform gelöst. Gemäß der Tabelle von Yu et al. haben sphärische TOPO-beschichtete CdSe (und damit in Annäherung auch BDT-beschichtete CdSe/ZnS) mit einem Absorptionsmaximum bei $518 \mathrm{~nm}$ einen Extinktionskoeffizienten von 70000 $\mathrm{M}^{-1} \mathrm{~cm}^{-1}$ und einen Durchmesser (inorganischer CdSe/ ZnS-Teil) von 2,5 nm. (Yu et al. 2003)

\section{Elektrodenpräparation}

Zur Reinigung wurden die Au-Elektroden (BASi, UK) mit $\mathrm{Al}_{2} \mathrm{O}_{3}$-Pulver mit abnehmender Korngröße (1 $\mu \mathrm{m}, 0.05$ $\mu \mathrm{m})$ jeweils 4 min poliert. Danach wurden sie voltammetrisch in $1 \mathrm{M} \mathrm{NaOH}(-800 \mathrm{mV}$ to $+200 \mathrm{mV}$ vs Ag/ $\mathrm{AgCl}, 1 \mathrm{M} \mathrm{KCl}$, Scanrate $300 \mathrm{mV} \mathrm{s}^{-1}$ ) und in 0,5 $\mathrm{M} \mathrm{H}_{2} \mathrm{SO}_{4}$ (-250 mV to $+1,75 \mathrm{~V}$ vs Ag/AgCl, $1 \mathrm{M} \mathrm{KCl,} \mathrm{Scanrate} 300$ $\mathrm{mV} \mathrm{s}^{-1}$ ) gereinigt. Zwischendurch wurden die Elektroden mit destilliertem Wasser abgespült und nach dem letzten Reinigungsschritt zusätzlich mit Ethanol.

Wenn nicht anders angegeben, wurden die Quantenpunkte zuerst mit einem Dithiol modifiziert und danach auf einer Goldelektrode immobilisiert (Au-[QDBDT]). Für den Ligandenaustausch wurden $5 \mu \mathrm{M}$ CdSe/ ZnS-Nanokristalle mit $100 \mathrm{mM}$ 1,4-Benzendithiol in Chloroform bei $40{ }^{\circ} \mathrm{C}$ und Schütteln für 3 Stunden inkubiert. Nach Zugabe von Methanol mit einem Volumenverhältnis von 1:1 wurde die Lösung bei $1000 \mathrm{U} /$ min für 9 min abzentrifugiert. Die Nanokristalle wurden in Toluen resuspendiert.

Frisch gereinigte Goldelektroden wurden mit Benzendithiol-beschichten CdSe/ZnS-Nanokristallen bei Raumtemperatur für 24 Stunden inkubiert. Die Quantenpunktlösung wurde in einem Eppendorfgefäß auf der Elektrode platziert. Die Elektroden wurden während der Inkubation geschüttelt. Nach der Inkubation wurden die Goldelektroden mit Toluen abgespült, um schwach gebundene Nanokristalle zu entfernen.

Für die Immobilisierung von TOPO-beschichteten CdSe/ZnS-Nanokristallen wurden die Goldelektroden zuerst mit Benzendithiol in Chloroform bei $40{ }^{\circ} \mathrm{C}$ für 3
Stunden inkubiert und mit Chloroform danach abgespült. Dann wurden die BDT-modifizierten Goldelektroden mit TOPO-beschichteten CdSe/ZnS-Nanokristalllösung in Chloroform bei Raumtemperatur für 24 Stunden inkubiert. Die Nanokristalllösung wurde auf der Elektrode platziert. Die Elektroden wurden während der Inkubation ebenfalls geschüttelt. Nach der Inkubation wurden die Goldelektroden mit Chloroform abgespült, um schwach gebundene Nanokristalle zu entfernen.

QCM-Chips (ICM, USA) mit einer Resonanzfrequenz von $10 \mathrm{MHz}$ (Durchmesser 5,1 mm) wurden mit Piranhalösung $\left(\mathrm{H}_{2} \mathrm{O}_{2}: \mathrm{H}_{2} \mathrm{SO}_{4} 1: 2 \mathrm{v} / \mathrm{v}\right)$ für $10 \mathrm{~min}$ gereinigt und mit destilliertem Wasser und Ethanol abgespült. Für die Modifizierung der QCM-Chips wurde die gleiche Prozedur verwendet wie für die Goldelektroden beschrieben. Die Inkubationsschritte wurde in einer Batchzelle durchgeführt.

Für die Modifikation des Elektrodensystems (Au[QD-BDT]) mit dem Mediator Pyrroloquinolinquinon (PQQ) wurde der Mediator an die BDT-modifizierten Quantenpunkte gekoppelt. Dafür wurden die Quantenpunkt-modifizierten Goldelektroden (Au-[QD-BDT]) mit 2,85 mM PQQ/ 10 mM EDC in 10 mM HEPES (N-[2Hydroxyethyl]piperazin-N'-[2-ethansulfonsäure]), pH 7 für 1,5 Stunden bei Raumtemperatur inkubiert. Danach wurden die Elektroden mit 100mM HEPES gewaschen.

\section{Messungen}

Größe und Konzentration der Quantenpunkte wurde mit Hilfe eines UV-vis-Spektrometers (Beckman Coulter, Dtl.) gemäß einer Kalibrationstabelle bestimmt. (Yu et al. 2003) Der Extinktionskoeffizient je Mol der Nanokristalle beim ersten exzitonischen Absorptionspeak für hochreine CdTe-, CdSe-und CdS-Nanokristalle ist stark abhängig von der Größe der Nanokristalle (quadratische bis kubische Abhängigkeit). (Yu et. al. 2003) Für die Messungen wurden Nanokristalle verwendet, die entweder durch überwachte Verfahren gereinigt oder durch kontrollierte Ätzmethoden präpariert wurden. Die Art der Oberflächenliganden, der Refraktionsindex des Lösungsmittels, die Photolumineszenz-Quantenausbeute der Nanokristalle, die für die Synthese verwendete Methode und die Temperatur bei den Messungen haben alle keinen detektierbaren Einfluss auf den Extinktionskoeffizient für eine bestimmte Nanokristallgröße innerhalb des experimentellen Fehlers.

QCM-Experimente wurden mit einem Multilab 3900 (J. Kitlička, Tscheschiche Republik) unter trockenen Bedingungen durchgeführt. 
Elektrochemische Experimente wurden mit einem Potentiostaten CHI 1205 (CH Instruments, USA) in einer selbstgebauten elektrochemischen Messzelle mit einer 3-Elektrodenanordnung durchgeführt. Eine Ag/AgCl-Elektrode (1M KCl, Microelectrodes Inc., USA) wurde als Referenzelektrode verwendet, ein Platindraht als Gegenelektrode. Das Volumen der Messzelle beträgt $2 \mathrm{ml}$. Gegenüber der Arbeitselektrode ist ein Lichtwellenleiter fixiert, der die Beleuchtung der ganzen Arbeitselektrode in einem definierten Abstand $(1 \mathrm{~cm})$ ermöglicht. Eine 150 W Xe-Bogenlampe von LOT Oriel (Darmstadt, Dtl.) wurde als Lichtquelle verwendet. Lichtpulse wurden manuell durch das Öffnen und Schließen einer Apertur erzeugt.

Für die elektrochemischen Messungen wurden Stammlösungen mit 200 mM NADH, 200 mM NAD $^{+}$ und $50 \mathrm{U}$ der Glukosedehydrogenase in $100 \mathrm{mM} \mathrm{HE}-$ PES-Puffer ( $\mathrm{pH} 8$ ) frisch prepariert. Eine Stammlösung mit 200 mM Glukose in 100 mM HEPES (pH 8) wurde einen Tag vor den Messungen präpariert und bei $5{ }^{\circ} \mathrm{C}$ gelagert, um eine Gleichgewicht der Mutarotation einzustellen. Kleine Mengen der Stammlösung wurden in die Messlösung injiziert, um die entsprechende Endkonzentration zu erhalten. Die Messungen wurden in 100 mM HEPES (pH8) unter Rühren durchgeführt.

\section{Ergebnisse und Diskussion}

Immobilisierung der Quantenpunkte

Für die Immobilisierung der CdSe/ZnS-Nanokristalle wurden deren organische Liganden aus der Synthese gegen 1,4-Benzendithiol (BDT) ausgetauscht. Die Verwendung eines kleinen Dithiols bietet zum einen die Möglichkeit, in einem ersten Schritt den ursprünglichen Liganden - Trioctylphosphinoxid (TOPO) - auszutauschen, zum anderen aber auch im zweiten Schritt die Bindung der Nanokristalle auf der Goldoberfläche der Elektrode über Chemisorption sicherzustellen (Au[QD-BDT]). Das Prinzipschema der Immobilisierung ist in Abbildung 1 dargestellt.

Der Nachweis für die erfolgreiche Abscheidung der Quantenpunkte auf der Elektrode erfolgt im einfachsten Fall durch eine Photostrommessung, wie in Abbildung 1 (II) gezeigt. Die Intensität und Richtung dieses Photostroms hängen vom angelegten Potential ab und sind ähnlich zu einem Quantenpunkt-Elektrodensystem, das schon für die Interaktion mit Cytochrom $c$ beschrieben wurde. (Stoll et al. 2008) Die Richtung des

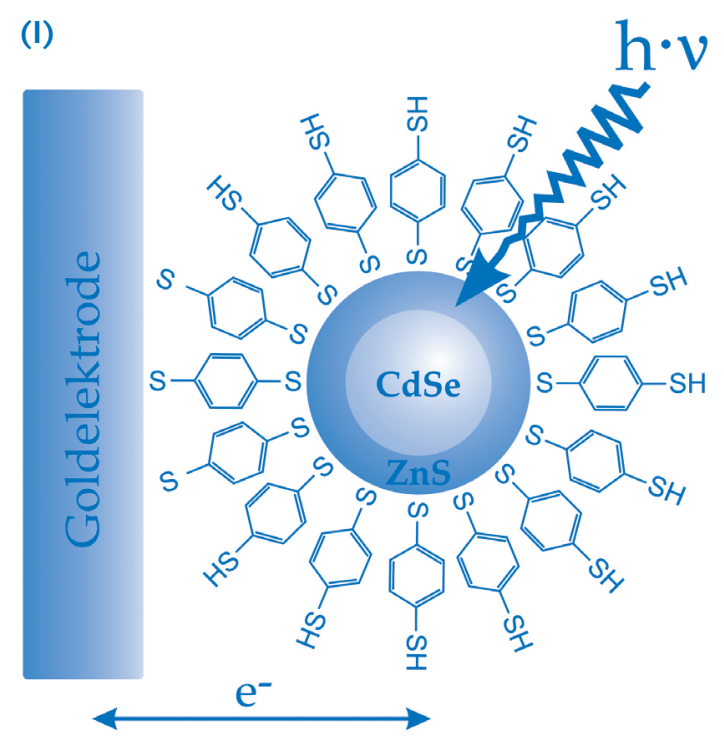

(II)

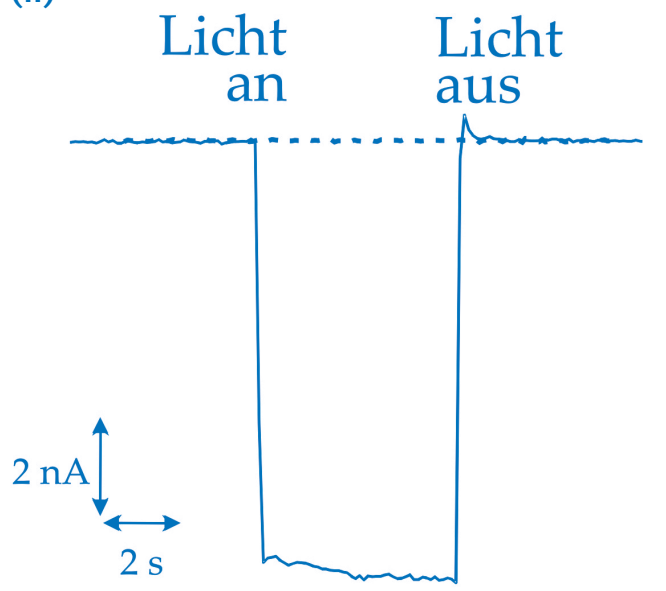

Abb. 1: (I) Prinzip der Immobilisierung der CdSe/ZnS-Quantenpunkte auf Gold über den Liganden 1,4-Benzendithiol (Au-[QD-BDT]); (II) Änderung der Stromantwort durch Beleuchtung der Elektrodenoberfläche, (---) unmodifizierte Goldelektrode, (-) Quantenpunkt-modifizierte Goldelektrode

Photostroms wechselt in Abhängigkeit des verwendeten Puffers und des $\mathrm{pH}$-Wertes bei einem Potential zwischen +100 und +300 mV (vs Ag/AgCl, $1 \mathrm{M} \mathrm{KCl)}$.

Um nachzuweisen, dass der Photostrom auf die Eigenschaften der Quantenpunkte zurückzuführen ist, wurde die Abhängigkeit des Stromes von der Wellenlänge des Lichtes untersucht. (Stoll et al. 2008) Der Photostrom der Quantenpunkt-modifizierten Elektrode folgt mit einem Peakstrom zwischen 520 und 540 nm eindeutig dem Absorptionsspektrum der CdSe/ZnSPartikel. Somit ist der gemessene Strom ein Resultat der Elektron-Loch-Paar-Generierung innerhalb der CdSe/ ZnS-Nanopartikel.

Es muss erwähnt werden, dass an einer blanken Goldelektrode kein Photostrom messbar ist. Jedoch wird nach der Quantenpunkt-Immobilisierung bei einem 
angelegten Potential von $+50 \mathrm{mV}$ vs $\mathrm{Ag} / \mathrm{AgC}, 1 \mathrm{M} \mathrm{KCl}$ ein negativer Photostrom in der Größenordnung von 8 nA beobachtet.

Die Stabilität des Photostroms wurde bei dem gleichen Potential getestet. Während der ersten Lichtpulse steigt der Photostrom an (siehe Abbildung 2) und bleibt danach fast stabil. Sogar nach 1 Stunde durchgehender Beleuchtung der Elektrodenoberfläche kann nur ein Abfall von etwa $10 \%$ festgestellt werden. Hier muss erwähnt werden, dass für die lichtgesteuerte Auslese eine Elektrodenbeleuchtung von 5 - 10 s ausreichend ist. Die beschriebene Immobilisierungsstrategie bietet somit in der getesteten Zeitperiode eine stabile photoschaltbare Schicht.

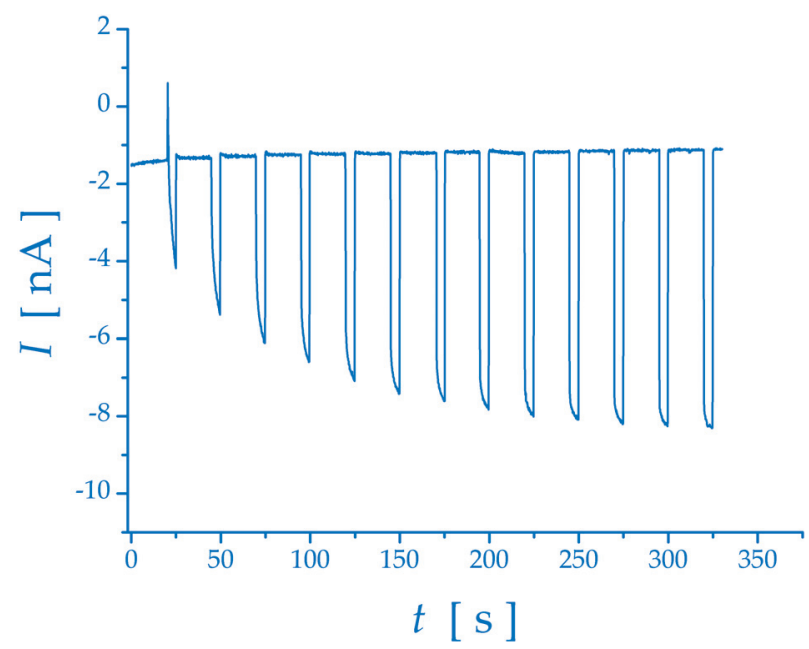

Abb. 2: Entwicklung der Photostromantwort von Au-[QD-BDT] für wiederholte Lichtpulse in 100 mM HEPES (N-[2-Hydroxyethyl]piperazin-N'[2-ethansulfonsäure]) pH8 bei $+50 \mathrm{mV}$ (vs Ag/AgCl, $1 \mathrm{M} \mathrm{KCl}$ )

Die Immobilisierung der Quantenpunkte wurde unabhängig durch QCM-Messungen (quartz crystal microbalance) verifiziert. Dafür wurden CdSe/ZnS-Nanokristalle auf dem Goldfilm des Quarzkristalls $\left(\mathrm{f}_{0}=10\right.$
$\mathrm{MHz})$ aufgebracht. Die Frequenzverschiebung beträgt etwa $-225 \mathrm{~Hz}$ mit einer Standardabweichung von $15 \%$ ( $n=6$ ) für die Immobilisierung der Quantenpunkte auf diesen Chips. Wenn wir eine ähnliche Situation für die Goldelektroden wie für QCM-Chips annehmen, lässt sich näherungsweise eine Oberflächenkonzentration der Quantenpunkte bestimmen. Das Molekulargewicht der CdSe/ZnS-Nanokristalle ist jedoch leider nicht genau bekannt, vor allem in Hinsicht darauf, dass die exakte Menge und Zusammensetzung der Oberflächenliganden noch nicht bestimmt wurde. Daher variieren die Werte zwischen verschiedenen Studien erheblich. (Komoto et al. 2006; Striolo et al. 2002) Wenn für die CdSe/ ZnS-Nanokristalle von einer Kugel mit 2,5 nm Durchmesser (ohne Ligand) und einer BDT-Schicht mit 10 20 Molekülen/nm² ausgegangen wird, kann anhand der Dichte von CdSe das Molekulargewicht mit etwa 50000 bis $80000 \mathrm{~g} \mathrm{~mol}^{-1}$ abgeschätzt werden. Mit einer Massenänderung von $4,4 \mathrm{ng} \mathrm{Hz}^{-1} \mathrm{~cm}^{-2}$ bei der QCM-Messung kann eine Oberflächenkonzentration im Bereich von 10 bis $20 \mathrm{pmol} \mathrm{cm}^{-2}$ und damit eine Bedeckung der Elektrode mit etwa einer Monolage bestimmt werden.

\section{Elektrokatalytische Oxidation von NADH}

In Anwesenheit von NADH kann eine deutliche Änderung des Photostroms beobachtet werden. Die NADHDetektion ist in einem relativ weiten Potentialfenster möglich. Für ein Elektrodenpotential, bei dem unter Beleuchtung kein Photostrom detektiert werden kann, erscheint nach Zugabe von NADH in die Messlösung ein anodischer Photostrom. Bei einem positiveren Potential erhöht sich der anodische Photostrom deutlich nach Zugabe von NADH (siehe Abbildung 3 (I)). Elektronen werden offensichtlich vom NADH zur angeregten Quantenpunktschicht unter Beleuchtung transportiert.

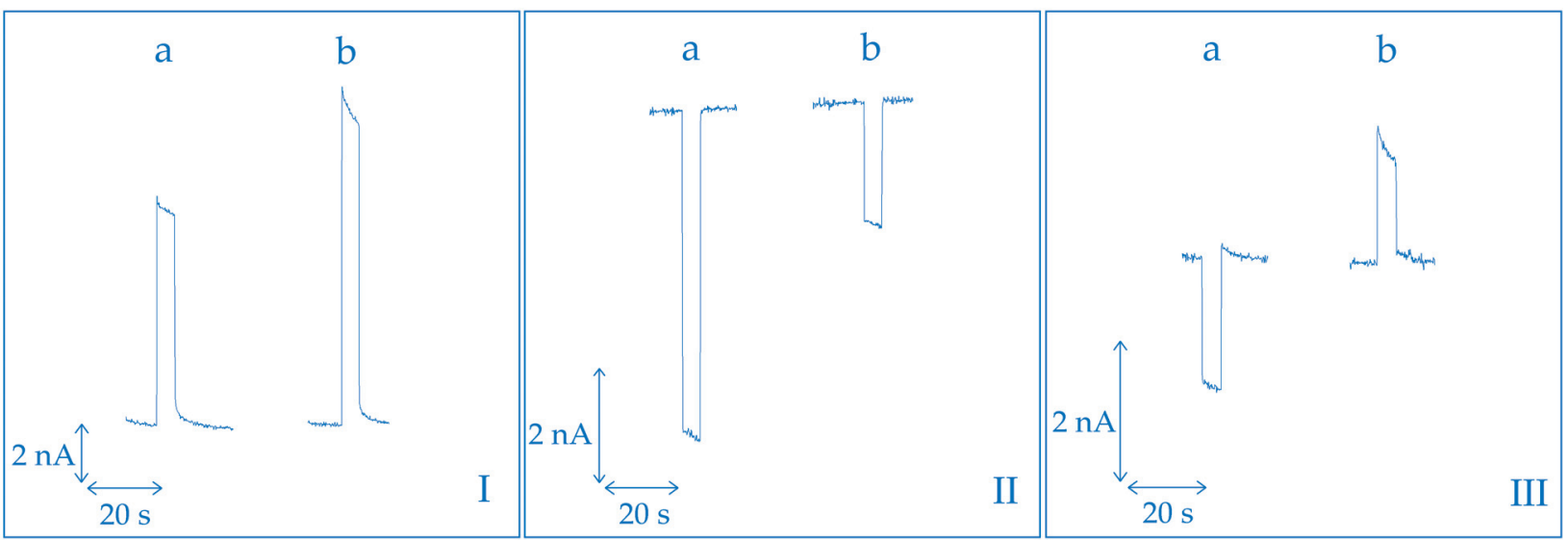

Abb. 3: Änderung des Photostroms für Au-[QD-BDT] durch Zugabe von NADH (a: ohne, b: mit 200 M NADH), 100 mM HEPES pH8; angelegte Potentiale waren bei (I) $+300 \mathrm{mV}$, (II) $+100 \mathrm{mV}$, (II) $+210 \mathrm{mV}$ (vs Ag/AgCl, $1 \mathrm{M} \mathrm{KCl}$ ) 
Bei Potentialen bei denen ein kathodischer Photostrom generiert wird (vor allem unterhalb $+100 \mathrm{mV}$ vs Ag/ $\mathrm{AgCl}, 1 \mathrm{M} \mathrm{KCl}$ ), verringert sich dieser Strom in Anwesenheit von NADH (siehe Abbildung 3 (II)). Das deutet daraufhin, dass Elektronen, die von der Elektrode zu den Nanokristallen übertragen werden, mit Elektronen vom NADH konkurrieren. Letztlich kann sogar bei einem entsprechenden Potential ein kathodischer Photostrom in einen anodischen übergehen, wie in Abbildung 3 (III) gezeigt.

In allen Fällen kann die Anwesenheit von NADH in der Lösung durch eine Änderung des Photostroms nachgewiesen werden. Im Bereich von $-100 \mathrm{mV}$ bis +200 mV (vs Ag/AgCl, $1 \mathrm{M} \mathrm{KCl)} \mathrm{kann} \mathrm{nur} \mathrm{eine} \mathrm{schwa-}$ che Abhängigkeit der Photostromänderung vom angelegten Potential festgestellt werden (siehe Abbildung 4). Bei negativeren Potentialen wird ein Ansteigen des kathodischen Photostroms beobachtet. Aufgrund der Konkurrenz des Elektronentransfers zwischen Elektrode bzw. NADH und der beleuchteten Nanokristallschicht wird die kinetische Situation komplizierter und deshalb anhand der vorliegenden Daten nicht weiter diskutiert.

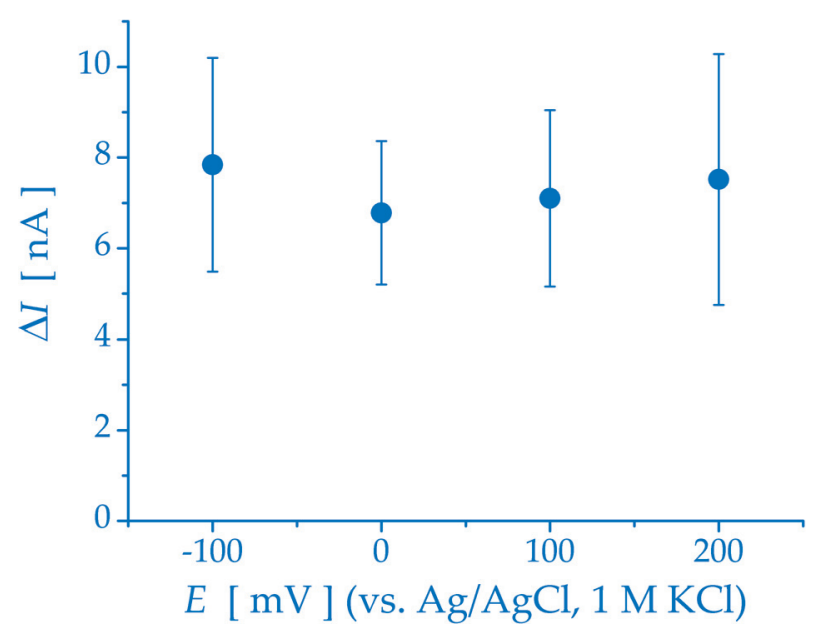

Abb. 4: Abhängigkeit der Photostromänderung vom angelegten Poten-

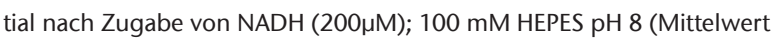
von 6 Elektroden, Au-[QD-BDT])

Für positivere Potentiale wird ebenfalls ein steigender Photostrom beobachtet. Da es jedoch Ziel bei der NADH-Oxidation ist, das Elektrodenpotential so niedrig wie möglich zu wählen und den elektrochemischen Stress für die Quantenpunkte zu minimieren, wurde die NADH-Detektion bei höheren Potentialen nicht weiter untersucht.

Im Potentialbereich von $-100 \mathrm{mV}$ bis $+200 \mathrm{mV}$ (vs $\mathrm{Ag} / \mathrm{AgCl}, 1 \mathrm{M} \mathrm{KCl}$ ) verursacht die NADH-Oxidation eine Photostromänderung, die in der gleichen Größen- ordnung liegt wie der Photostrom, der in Abwesenheit von NADH entsteht. Die nur schwache Abhängigkeit der Photostromänderung vom angelegten Potential an der Elektrode zeigt, dass die NADH-Oxidation nicht einfach über dieses Potential verbessert werden kann. Die NADH-Oxidation wird offensichtlich vorwiegend durch die katalytischen Eigenschaften der Nanopartikel verursacht.

Es muss ebenfalls erwähnt werden, dass die Nanopartikel-modifizierte Elektrode nicht für die Detektion von $\mathrm{H}_{2} \mathrm{O}_{2}$ - einem anderen potentiellen Produkt von Oxidoreduktase-Reaktionen - verwendet werden kann. Die Nanopartikel bieten keine geeignete Oberfläche für die Oxidation dieses enzymatischen Nebenproduktes.

Da die Änderung des Photostroms auf die elektrochemische Reaktion von NADH an der Quantenpunktmodifizierten Elektrode zurückzuführen ist, kann ein solches Nanopartikel-basiertes System effektiv für die Detektion von NADH eingesetzt werden und bietet damit das Fundament für die Konstruktion eines Sensorsystems. Im Folgenden wurden alle Messungen bei $+50 \mathrm{mV}$ (vs Ag/AgCl, $1 \mathrm{M} \mathrm{KCl}$ ) durchgeführt, um große Hintergrundsignale in Abwesenheit des Analyten zu vermeiden.

Sensitivität für NADH kann in einem Bereich von $20 \mu \mathrm{M}$ bis $2 \mathrm{mM}$ erreicht werden, wie in Abbildung 5 (II) zu sehen. Die Konzentrationsabhängigkeit zeigt dabei ein Sättigungsverhalten. Damit verhält es sich ähnlich wie die Photostromänderungen an Mercaptopropionsäure-modifizierten CdSe/ZnS-Quantenpunkt-Elektroden in Anwesenheit von Cytochrom c. (Stoll et al. 2008)

Kontrollexperimente bestätigen, dass der Nanopartikelligand Benzendithiol nicht als Mediator am Elektronentransferprozess von NADH zu den CdSe/ZnS-Quantenpunkten beteiligt zu sein scheint. Goldelektroden, die nur mit Benzendithiol beschichtet wurden, zeigen nur einen sehr schwachen Photostrom (weniger als - $1 \mathrm{nA}$ bei $+50 \mathrm{mV}$ vs Ag/AgCl, $1 \mathrm{M} \mathrm{KCl}$ ) und keine nennenswerte Änderung in Anwesenheit von 1 mM NADH.

Um den Einfluss der Nanopartikeloberflächen-Modifizierung zu untersuchen, sind etwas andere Elektroden präpariert worden, wie in Abbildung 5 (I) gezeigt. Goldelektroden werden zuerst mit Benzendithiol modifiziert und im zweiten Schritt mit CdSe/ZnS-Nanokristallen beschichtet (Au-BDT-[QD/TOPO]). Das heißt, der ursprüngliche Ligand der Quantenpunkte - Trioctylphosphinoxid (TOPO) - ist immer noch auf der Oberfläche der Nanopartikel und in Kontakt mit der Lösung. Solch ein Elektrodensystem zeigt ein ähnliches Verhalten der 
(I)

$\mathbf{a}$

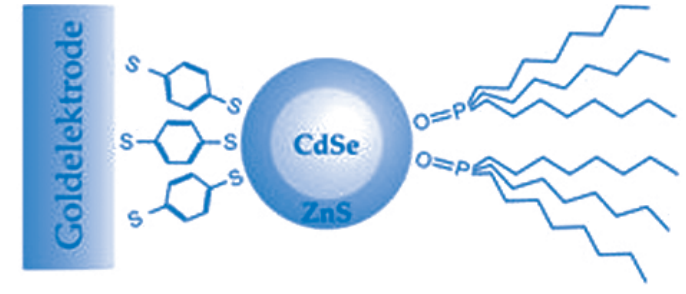

b

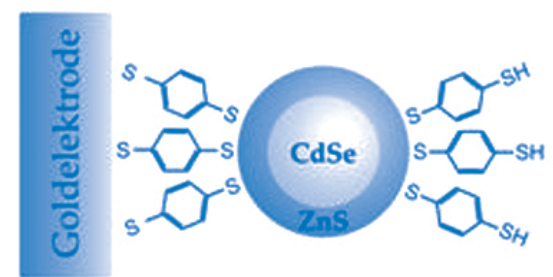

(II)

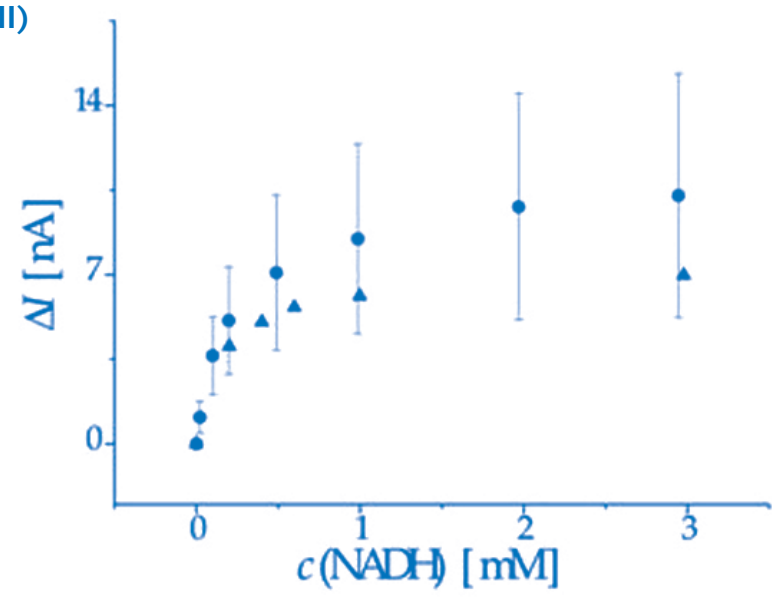

Abb. 5: (I) Schema von zwei verschiedenen Elektrodensystemen (a) Au-BDT-[QD/TOPO], (b) Au-[QD-BDT]; (II) Photostromänderung für zwei verschiedene Elektrodensysteme nach Zugabe von NADH; (•) für (a), (^) für (b), in 100 mM HEPES pH8 bei +50 mV vs Ag/AgCl, $1 \mathrm{M} \mathrm{KCl} \mathrm{(Mittelwert} \mathrm{von} 3$ Elektroden)

Photostromänderung in Anwesenheit von NADH im Vergleich zu Elektroden mit Nanopartikeln, deren Liganden zuerst gegen Benzendithiol ausgetauscht wurden (AU-[QD-BDT]), wie in Abbildung 5 (II) zu sehen.

Das deutet auf einen direkten Elektronentransfer von NADH auf die CdSe/ZnS-Nanopartikel hin. Es kann jedoch nicht gänzlich ausgeschlossen werden, dass die Elektronentransferreaktion durch BDT wegen seiner aromatischen Struktur unterstützt wird (vor allem unter Beleuchtung).

Es wurde ebenfalls untersucht, ob die NADH-Detektion durch einen bekannten Mediator für die NADH-Elektrochemie - Pyrroloquinolinquinon (PQQ) - verbessert werden kann. (Katz et al. 1994) Dazu wurden die Quantenpunkte zusätzlich nach der Immobilisierung auf der Goldelektrode mit PQQ modifiziert. Jedoch kann keine nennenswerte Verstärkung der Photostromänderung im Vergleich zu PQQ-freien Au-CdSe/ZnS-Elektroden festgestellt werden. Somit führt die Verwendung dieses oberflächengebundenen Mediators zu keinem Fortschritt und ist nicht notwendig für die elektrokatalytische Detektion von NADH.

\section{Kombination mit einer Dehydrogenasereaktion}

NADH ist als Kofaktor in viele Dehydrogenase-katalysierte Reaktionen involviert. Die Konzentration von NADH kann wie zuvor beschrieben durch Photostrommessungen bestimmt werden. Somit kann das QuantenpunktElektrodensystem zur Verfolgung solcher katalytischen Reaktionen verwendet werden. Die Menge an NADH, die in einer enzymatischen Katalyse produziert wird, hängt direkt von der Konzentration des Substrates ab.

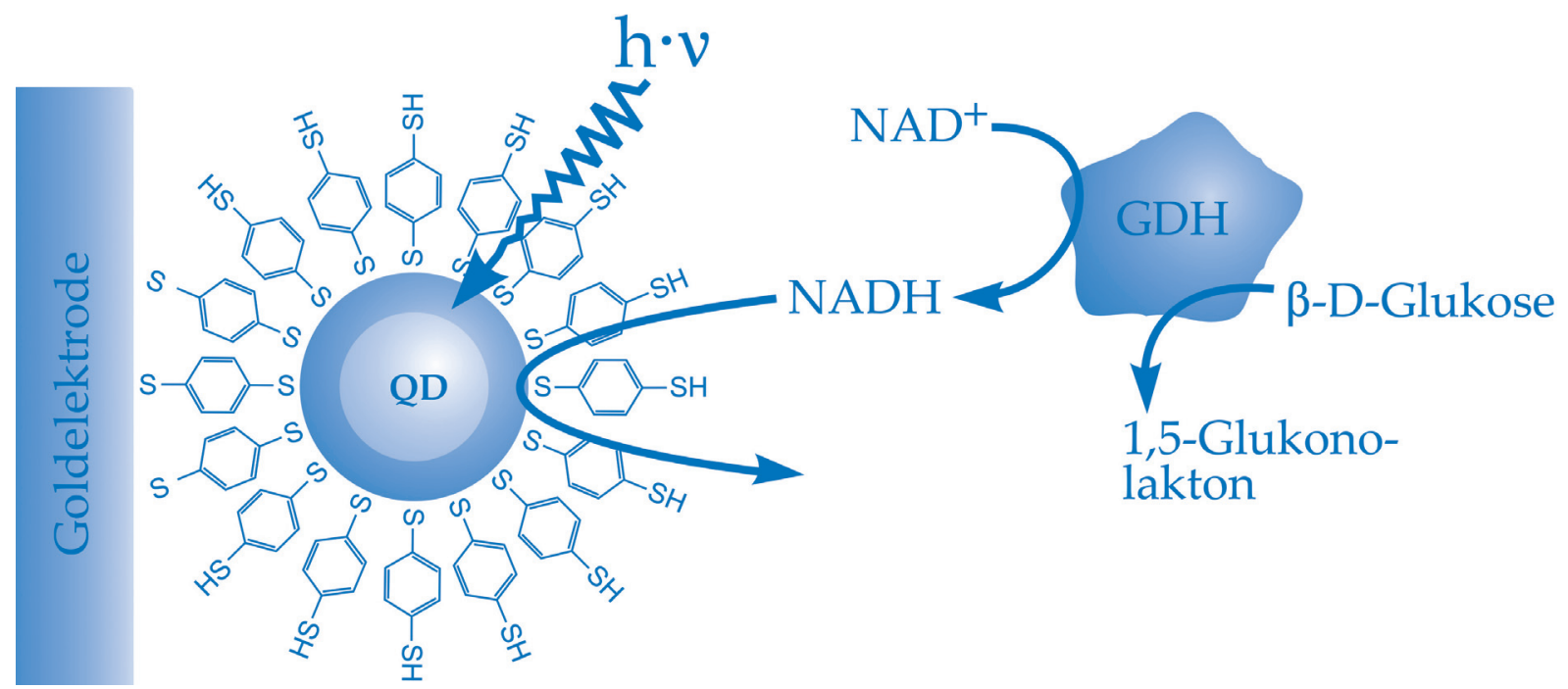

Abb. 6: Schema der Glukose-Detektion an einer Quantenpunkt-modifizierten Goldelektrode über die katalytische Produktion von NADH durch das Enzym Glukosedehydrogenase (GDH) in Lösung 
Um die Realisierbarkeit dieses Konzepts zu demonstrieren, wurde Glukosedehydrogenase als Biokatalysator gewählt. Diese Dehydrogenase katalysiert die Reaktion von $\beta$-D-Glukose zu D-Gluconolacton, während der Kofaktor $\mathrm{NAD}^{+} \mathrm{zu}$ NADH reduziert wird. Dieses Prinzip ist in Abbildung 6 dargestellt.

Photostrommessungen mit Quantenpunkt-modifizierten Elektroden vor und nach Zugabe von Glukose ohne Enzym zeigen keine Änderung des Signals im getesteten Potentialbereich von $-100 \mathrm{mV}$ bis $+200 \mathrm{mV}$ (vs $\mathrm{Ag} / \mathrm{AgCl}, 1 \mathrm{M} \mathrm{KCI})$, exemplarisch in Abbildung 7 dargestellt.

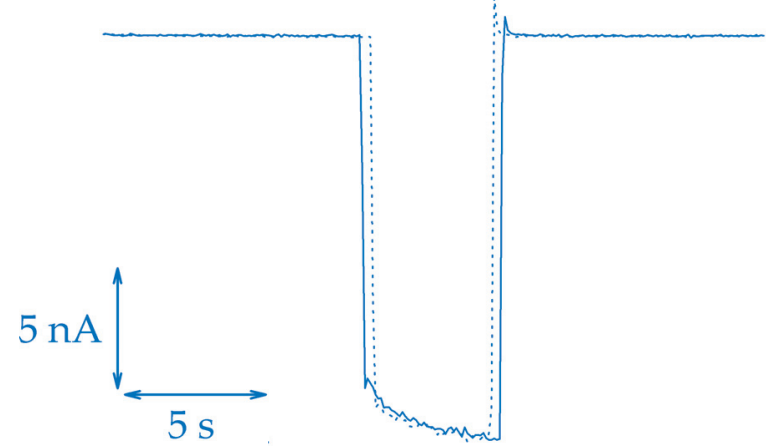

Abb. 7: Photostromantwort einer Quantenpunkt-modifizierten Elektrode ohne das Enzym GDH; (...) ohne und (-) in Anwesenheit von $500 \mu \mathrm{M}$ Glukose in $100 \mathrm{mM}$ HEPES pH8 bei $+50 \mathrm{mV}$ (vs Ag/AgCl, $1 \mathrm{M} \mathrm{KCl}$ )

Das zeigt, dass Glukose nicht direkt an Quantenpunkt-modifizierten Goldelektroden oxidiert wird. Jedoch kann in Anwesenheit des Enzyms Glukosedehydrogenase und seines Kofaktors $\mathrm{NAD}^{+}$eine konzentrationsabhängige Änderung des Photostroms mit dem hier entwickelten Nanopartikel-basierten System detektiert werden, wie in Abbildung 8 gezeigt. Das Glu-

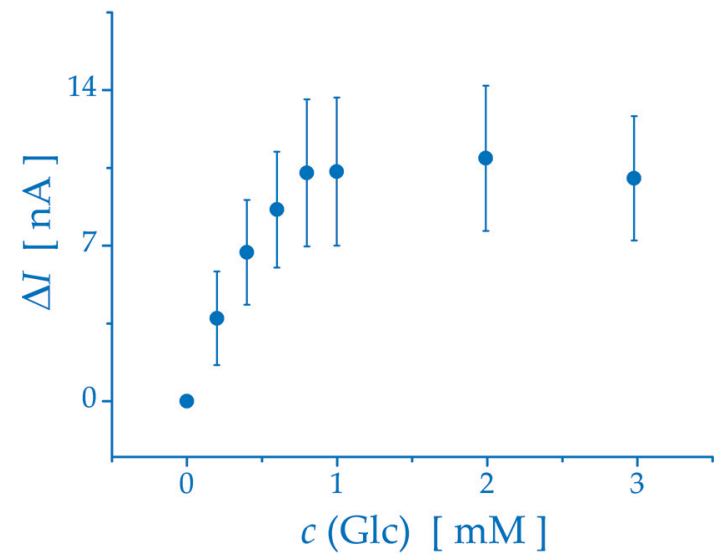

Abb. 8: Änderung des Photostroms einer Quantenpunkt-modifizierten Elektrode (Au-[QDBDT]) nach Zugabe von Glukose in Anwesenheit von $50 \mathrm{U}$ Glukosedehydrogenase und $1 \mathrm{mM} \mathrm{NAD}^{+}$in 100m M HEPES pH8 bei $+50 \mathrm{mV}$ (vs Ag/ $\mathrm{AgCl}, 1 \mathrm{M} \mathrm{KCl}$ ), Mittelwert von 4 Elektroden kosesignal wird über den Elektronentransfer über das Enzym zu NADH konvertiert, und NADH wird schließlich durch den Elektronentransfer zu den beleuchteten Quantenpunkten detektiert. Der Konzentrationsbereich für die Glukose-Detektion ist ähnlich dem der NADH-Detektion. Die Sensitivität für Glukose wird bis etwa $1 \mathrm{mM}$ beobachtet. Zusammenfassend kann gesagt werden, dass durch die Kombination eines spezifischen Biokatalysators und einer Quantenpunkt-modifizierten Elektrode eine bioanalytische Signalkette konstruiert werden kann, die durch Beleuchtung der Sensoroberfläche ein-und ausschaltbar ist.

\section{Fazit}

Es wurde eine nanostrukturierte Elektrode auf der Basis von CdSe/ZnS-Quantenpunkten für die sensitive Messung des Enzym-Kofaktors NADH entwickelt. Zum ersten Mal kann die lichtgesteuerte Auslese von NADHKonzentrationen in Lösung mit diesem System gezeigt werden.

Die Immobilisierung der halbleitenden Nanopartikel über ein Dithiol auf Goldelektroden kann durch die Aufnahme von Photoströmen nachgewiesen werden. Durch Photoanregung werden Elektron-Loch-Paare in den halbleitenden Nanokristallen gebildet, was bei Polarisierung der Elektrode zu einem Ladungstransfer führt. In Abhängigkeit des angelegten Potentials können anodische oder kathodische Photoströme detektiert werden. Die Nanokristallabscheidung kann ebenfalls durch QCM-Messungen (quartz crystal mircobalance) verifiziert werden. Die Frequenzänderung deutet auf eine Bedeckung der Elektrodenoberfläche mit etwa einer Monolage der CdSe/ZnS-Nanopartikel hin.

In Anwesenheit von NADH wird eine Änderung des Photostroms beobachtet, abhängig von der NADHKonzentration. Die Detektion ist in einem Bereich von $20 \mu \mathrm{M}$ bis $2 \mathrm{mM}$ möglich. Es muss ebenfalls hervorgehoben werden, dass für die NADH-Oxidation nur ein relativ geringes Elektrodenpotential mit diesem Nanopartikel-basierten System angelegt werden muss.

Es wurde ebenfalls gezeigt, dass CdSe/ZnS-Nanokristall-modifizierte Goldelektroden für die lichtgesteuerte Analyse von Substraten verwendet werden können, die in NADH-produzierenden Enzymreaktionen verbraucht werden. Dies konnte für die Detektion von Glukose mit Hilfe von Glukosedehydrogenase als Beispiel demonstriert werden. 


\section{Danksagung}

Dieses Projekt wird durch die Deutsche Forschungsgemeinschaft finanziell unterstützt (Projekt Li 706/ 2-1, PA 794/3-1). Weiterer Dank gilt Herrn Schäfer (TH Wildau [FH]) für die Bearbeitung der Abbildungen.

\section{Literatur}

Blaedel, W. J.; Jenkins, R. A. (1975): Electrochemical oxidation of reduced nicotinamide adenine dinucleotide. Analytical Chemistry, 47, 1337-1343.

Brus, L. (1991): Quantum Crystallites and Nonlinear Optics. Applied Physics A: Materials Sciences \& Processing, 53, 465-474.

Chan, W. C. W.; Maxwell, D. J.; Gao, X. H.; Bailey, R. E.; Han, M. Y.; Nie, S. M. (2002): Luminescent quantum dots for multiplexed biological detection and imaging. Current Opinion Biotechnology, 13, 40-46.

Dabbousi, B. O.; Rodriguez Viejo, J.; Mikulec, F. V.; Heine, J. R.; Mattoussi, H.; Ober, R.; Jensen, K. F.; Bawendi, M. G. (1997): (CdSe)ZnS Core-Shell Quantum Dots: Synthesis and Characterization of a Size Series of Highly Luminescent Nanocrystallites. Journal of Physical Chemistry B, 101, 9463-9475.

Freeman, R.; Gill, R.; Beissenhirtz, M.; Willner, I. (2007): Self-assembly of semiconductor quantumdots on electrodes for photoelectrochemical biosensing. Photochemistry \& Photobiology Sciences, 6, 416-422.

Fukuzumi, S.; Nishizawa, N.; Tanaka, T. (1984): Mechanism of Hydride Transfer from an NADH Model Compound to $\mathrm{p}$-Benzoquinone Derivatives. The Journal of Organic Chemistry, 49, 35713578.

Gerion, D.; Chen, F. Q.; Kannan, B.; Fu, A. H.; Parak, W. J.; Chen, D. J.; Majumdar, A.; Alivisatos, A. P.: Room-Temperature SingleNucleotide Polymorphism and Multiallele DNA Detection Using Fluorescent Nanocrystals and Microarrays. Analytical Chemistry, $75,4766-4772$.

Gill, R.; Zayats, M.; Willner, I. (2008): Halbleiterquantenpunkte für die Bioanalyse. Angewandte Chemie, 120, 7714-7736; Angewandte Chemie. International Edition, 47, 7602-7625.

Goldman, E. R.; Clapp, A. R.; Anderson, G. P.; Uyeda, H. T.; Mauro, J. M.; Medintz, I. L.; Mattoussi, H. (2004): Multiplexed Toxin Analysis Using Four Colors of Quantum Dot Fluororeagents. Analytical Chemistry, 76, 684-688.

Gorton, L. (1986): Chemically Modified Electrodes for the Electrocatalytic Oxidation of Nicotinamide Coenzymes. Journal of the Chemical Society, Faraday Transactions, 82, 1245-1258.

Jaegfeldt, H. (1981): A Study of the Products Formed in the Electrochemical Reduction of Nicotinamide-Adenine-Dinucleotide. Bioelectrochemistry and Bioenergetics, 8, 355-370.

Katakis, I.; Dominguez, E. (1997): Catalytic Electrooxidation of NADH for Dehydrogenase Amperometric Biosensors. Mikrochimica Acta, 126, 11-32.

Katz, E.; Lotzbeyer, T.; Schlereth, D. D.; Schuhmann, W.; Schmidt, H. L. (1994): Electrocatalytic oxidation of reduced nicotinamide coenzymes at gold and platinum electrode surfaces modified with a monolayer of pyrroloquinoline quinone. Effect of $\mathrm{Ca} 2+$ cations. Journal of Electroanalytical Chemistry, 373, 189-200.

Katz, E., Willner, I.; Wang, J. (2004): Electroanalytical and Bioelectroanalytical Systems Based on Metal and Semiconductor Nanoparticles. Electroanalysis, 16(1-2), 19-44.
Katz, E.; Zayats, M.; Willner, I.; Lisdat, F. (2006): Controlling the direction of photocurrents by means of CdS nanoparticles and cytochrome c-mediated biocatalytic cascades. Chemical Communications, 1395-1397.

Klostranec, J. M.; Chan, W. C. W. (2006): Quantum Dots in Biological and Biomedical Research: Recent Progress and Present Challenges. Advanced Materials, 18, 1953-1964.

Komoto, A.; Maenosono, S. (2006): Photoinduced fluorescence intensity oscillation in a reaction-diffusion cell containing a colloidal quantum dot dispersion. Journal of Chemical Physics, 125, DOI 10.1063/1.2338804.

Lau, C.; Flechsig, G. U.; Grundler, P.; Wang, J. (2005): Electrochemistry of nicotinamide adenine dinucleotide (reduced) at heated platinum electrodes. Analytica Chimica Acta, 554, 74-78.

Lin, C. A. J.; Liedl, T.; Sperling, R. A.; Fernandez-Arguelles, M. T.; CostaFernandez, J. M.; Pereiro, R.; Sanz-Medel, A.; Chang, W. H.; Parak, W. J. (2007): Bioanalytics and biolabeling with semiconductor nanoparticles (quantum dots). Journal of Materials Chemistry, 17, 1343-1346.

Lisdat, F.; Dronov, R.; Möhwald, H.; Scheller, F. W.; Kurth, D. G. (2009): Self-assembly of electroactive protein architectures on electrodes for the construction of biomimetic signal chains. Chemical Communications, 274-283.

Munteanu, F. D.; Mano, N.; Kuhn, A.; Gorton, L. (2002): Mediatormodified electrodes for catalytic NADH oxidation: high rate constants at interesting overpotentials. Bioelectrochemistry, $56,67-72$.

Nagy, G.; Kapui, I.; Gorton, L. (1995): Effect of surfactants on the signal of chemically modified amperometric electrodes. Sensors and Actuators, B, 24, 323-327.

Pardo-Yissar, V.; Katz, E.; Wasserman, J.; Willner, I. (2003): Acetylcholine Esterase-Labeled CdS Nanoparticles on Electrodes: Photoelectrochemical Sensing of the Enzyme Inhibitors. Journal of the American Chemical Society, 125, 622-623.

Pellegrino, T.; Kudera, S.; Liedl, T.; Javier, A. M.; Manna, L.; Parak, W. J. (2005): On the Development of Colloidal Nanoparticles towards Multifunctional Structures and their Possible Use for Biological Applications. Small, 1, 48-63.

Persson, B.; Gorton, L. (1990): A comparative study of some 3,7-diaminophenoxazine derivatives and related compounds for electrocatalytic oxidation of NADH, Journal of Electroanalytical Chemistry, 292, 115-138.

Prodromidis, M. I.; Karayannis, M. I. (2002): Enzyme Based Amperometric Biosensors for Food Analysis. Electroanalysis, 14, 241-261.

Raj, C. R.; Jena, B. K. (2005): Efficient electrocatalytic oxidation of NADH at gold nanoparticles self-assembled on three-dimensional sol-gel network. Chemical Communications, 2005-2007.

Reiss, P.; Bleuse, J.; Pron, A. (2002): Highly Luminescent CdSe/ZnSe Core/Shell Nanocrystals of Low Size Dispersion. Nano Letters, 2, 781-784.

Stoll, C.; Gehring, C.; Schubert, K.; Zanella, M.; Parak, W. J.; Lisdat, F. (2008): Photoelectrochemical signal chain based on quantum dots on gold-Sensitive to superoxide radicals in solution. Biosensors and Bioelectronics, 24, 260-265.

Stoll, C.; Kudera, S.; Parak, W. J.; Lisdat, F. (2006): Quantum Dots on Gold: Electrodes For Photoswitchable Cytochrome c Electrochemistry. Small, 2, 741-743. 
Striolo, A.; Ward, J.; Prausnitz, J. M.; Parak, W. J.; Zanchet, D.; Gerion, D.; Milliron, D.; Alivisatos, A. P. (2002): Molecular Weight, Osmotic Second Virial Coefficient, and Extinction Coefficient of Colloidal CdSe Nanocrystals. Journal of Physical Chemistry B, $106,5500-5505$.

Wang, J. (2005): Nanomaterial-Based Amplified Transduction of Biomolecular Interactions. Small, 1(11), 1036-1043.

Willner, I.; Basnar, B.; Willner, B. (2007): Nanoparticle-enzyme hybrid systems for nanobiotechnology. FEBS Journal, 274, 302-309.

Willner, I.; Patolsky, F.; Wasserman, J. (2001): Photoelectrochemistry with Controlled DNA-Cross-Linked CdS Nanoparticle Arrays. Angewandte Chemie International Edition, 40(10), 1861-1864.

Xiao, Y. H.; Li, C. M. (2008): Nanocomposites: From Fabrications to Electrochemical Bioapplications. Electroanalysis, 20(6), 648-662.

Yu, W. W.; Qu, L. H.; Guo, W. Z.; Peng, X. G. (2003): Experimental Determination of the Extinction Coefficient of CdTe, CdSe, and CdS Nanocrystals. Chemistry of Materials, 15, 2854-2860.

\section{Autoren}

\section{Kirsten Schubert, M.Sc.}

TH Wildau [FH]

Biosystemtechnik

kirsten.schubert@tfh-wildau.de

Prof. Dr. Fred Lisdat

TH Wildau [FH]

Biosystemtechnik

fred.lisdat@tfh-wildau.de

Waqas Khalid, M.Sc.

Zhao Yue, M.Sc.

Prof. Dr. Wolfgang J. Parak

Philipps-Universität Marburg

Biophotonik

Renthof 7

35037 Marburg 